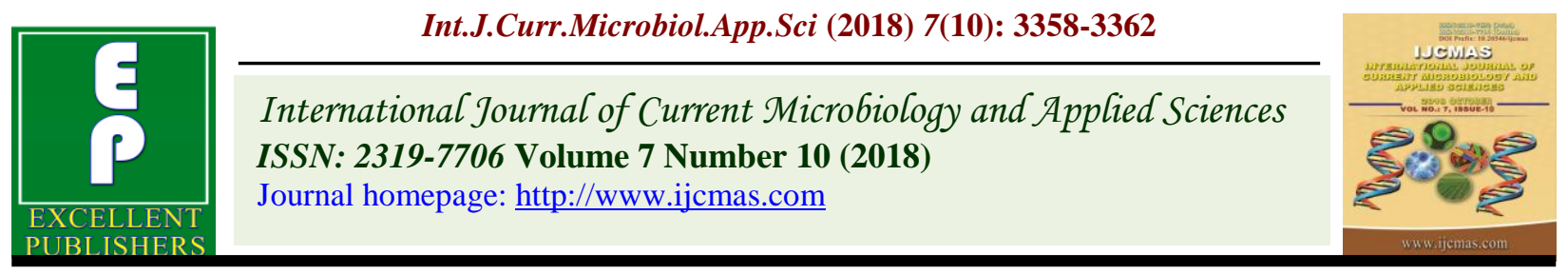

Original Research Article

https://doi.org/10.20546/ijcmas.2018.710.389

\title{
Impact of Robust Estimators on Variance Estimation in Survey Sampling, Using Conventional and Non-Conventional Parameters as Auxiliary Information
}

\author{
M.A. Bhat ${ }^{*}$ T.A. Raja and S. Maqbool \\ Division of Agricultural Economics and Statist SKUAST-Kashmir (190025), India \\ *Corresponding author
}

\section{A B S T R A C T}

Keywords

Sample Random

Sampling, Bias, MSE,

Quartiles and efficiency

Article Info

Accepted:

24 September 2018

Available Online:

10 October 2018
In the present study, we have developed new estimators for the estimation of finite population variance by using auxiliary information as combination of conventional and non-conventional measures. Bias and mean square error has been worked out up to the first order of approximation. The empirical study has been carried out through numerical demonstration, under which improved estimators have performed better than the other existing estimators.

\section{Introduction}

Variance estimation has great scope in the fields like medical sciences, Agricultural and Horticultural sciences, Biological sciences and Industries etc. In the field of medical sciences, a physician needs to have the knowledge about the levels of variations like pulse rate, body temperature and blood pressure to prescribe the suitable treatment. Similarly an agriculturalist needs the knowledge about the levels of variations like soil fertility variation, genetic variations and climatic variations to plant the crop. Thus variations are present everywhere in our day to day life. In order to obtain the precise and valid estimates, we have proposed new estimators for the estimation of finite population variance. Various authors have generalized many estimators such as Isaki (1983), who introduced the ratio and regression estimator, Kadilar and Cingi (2006a), who searched the estimators by utilizing the coefficient of skewkness as supplementary information to enhance the efficiency of estimator. Upadhya and Singh (1999) incorporated the coefficient of kurtosis as auxiliary information to improve the efficiency of estimators over existing estimators. Similarly Bhat et al., (2018) have used linear combination of skewness and quartiles as auxiliary information to obtain the precision of estimators. Sarginder Singh (2003), Advance Sampling theory with Applications (2003), page (1135-1136). 
In our present study, we have incorporated the combination of conventional and nonconventional parameters to accelerate the efficiency of proposed estimators.

Let the finite population under survey be $U=\left\{U_{1}, U_{2}, \ldots, U_{N}\right\}$, consists of $\mathrm{N}$ distinct and identifiable units. Let $\mathrm{Y}$ be a real variable with value $\mathrm{Y}_{\mathrm{i}}$ measured on $U_{i}, i=1,2,3 \ldots \ldots . . . N$, giving a vector $Y=\left\{y_{1}, y_{2}, \ldots, y_{N}\right\}$. The goal is to estimate the populations mean $\bar{Y}=\frac{1}{N} \sum_{I=1}^{N} y_{i}$ variance $S_{Y}^{2}=\frac{1}{N-1} \sum_{I=1}^{N}\left(y_{i}-\bar{y}\right)^{2}$ on the basis of random sample selected from a population U. In this paper, our aim is to estimate the precise and reliable estimates for finite population variance when the population under survey is non-normal or skewed.

\section{Materials and Methods}

\section{Notations}

$N=$ population size $n=$ sample size .

$\gamma=\frac{N-n}{N n}$

$Y=$ Study Variable, $\mathrm{X}=$ Auxiliary Variable,

$\bar{X}, \bar{Y}=$ population means

$\bar{x}, \bar{y}=$ Sample means

$S_{Y}^{2}, S_{x}^{2}=$ population Variances,

$s_{y}^{2}, s_{x}^{2}=$ Sample variances

$C_{x}, C_{y}=$ Cofficient of variations,

$\rho=$ Cofficient of Correlation,

$\beta_{1(x)}=$ Cofficient of Skewness

$\beta_{2(x)}=$ Cofficient of kurtosis

$\beta_{2(y)}=$ Cofficient of kurtosis,
$Q_{1}=$ first quartile, $Q_{2}=\sec$ ond quartile, $Q_{a}=$ quartile average, $Q_{d}=$ quartile deviation and TM $=$ Tri-mean, $\lambda_{r s}=\frac{\mu_{r s}}{\mu_{r s}^{\frac{r}{2}} \mu_{r s}^{\frac{s}{2}}}, \mu_{r s}=\frac{1}{N-1} \sum_{i=1}^{N}(Y-\bar{Y})^{r}(X-\bar{X})^{s}$

Existing Estimators from the literature

Ratio type Variance estimator proposed by Isaki (1983)

$$
\hat{S}_{R}^{2}=s_{y}^{2} \frac{S_{x}^{2}}{S_{x}^{2}}
$$

$\operatorname{Bias}\left(\left(\hat{S}_{R}^{2}\right)=\gamma S_{y}^{2}\left[\left(\beta_{2(x)}-1\right)-\left(\lambda_{22}-1\right)\right]\right.$

MSE

$\left(\left(\hat{S}_{R}^{2}\right)=\right.$

$\gamma S_{y}^{4}\left[\left(\beta_{2(y)}-1\right)+\left(\beta_{2(x)}-1\right)-2\left(\lambda_{22}-1\right)\right]$

Ratio type Variance estimator proposed by Kadilar and Cingi (2006a)

$$
\hat{S}_{k c 1}^{2}=s_{y}^{2}\left[\frac{S_{x}^{2}+C_{x}}{s_{x}^{2}+C_{x}}\right]
$$

Bias

$\left(\left(\hat{S}_{k c 1}^{2}\right)=\gamma S_{y}^{2} A_{1}\left[A_{1}\left(\beta_{2(x)}-1\right)-\left(\lambda_{22}-1\right)\right]\right.$

MSE

$$
\begin{aligned}
& \left(\begin{array}{l}
\left(\hat{S}_{k c 1}^{2}\right)= \\
\gamma S_{y}^{4}\left[\left(\beta_{2(y)}-1\right)+A_{1}^{2}\left(\beta_{2(x)}-1\right)-2 A_{1}\left(\lambda_{22}-1\right)\right]
\end{array}\right.
\end{aligned}
$$

Ratio type variance estimator proposed by Upadhya and Singh (1999)

$$
\hat{S}_{U S}^{2}=s_{y}^{2}\left[\frac{S_{x}^{2}+\beta_{2 x}}{s_{x}^{2}+\beta_{2 x}}\right]
$$

Bias

$\left(\hat{S}_{U S}^{2}\right)=\gamma S_{y}^{2} A_{U S}\left[A_{U S}\left(\beta_{2(x)}-1\right)-\left(\lambda_{22}-1\right)\right]$ 
MSE

$\left(\hat{S}_{U S}^{2}\right)=$

$\gamma S_{y}^{4}\left[\left(\beta_{2(y)}-1\right)+A_{U S}^{2}\left(\beta_{2(x)}-1\right)-2 A_{U S}\left(\lambda_{22}-1\right)\right]$

New improved estimators

$$
\begin{aligned}
& \hat{S}_{M S_{1}}^{2}=s_{y}^{2}\left[\frac{S_{x}^{2}+(\bar{X} \times T M)}{s_{x}^{2}+(\bar{X} \times T M)}\right], \\
& \hat{S}_{M S_{2}}^{2}=s_{y}^{2}\left[\frac{S_{x}^{2}+\left(\bar{X} \times Q_{d}\right)}{s_{x}^{2}+\left(\bar{X} \times Q_{d}\right)}\right]
\end{aligned}
$$

We have derived here the bias and mean square error of the proposed estimator $\hat{S}_{M S i}^{2} ; i=1,2$ to first order of approximation as given below

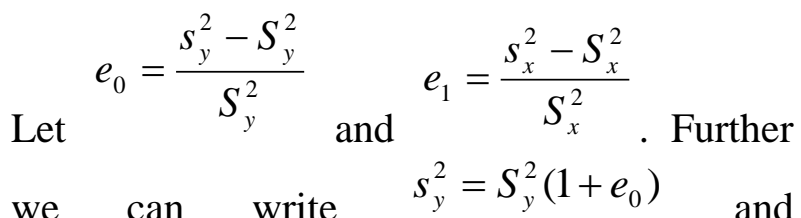
$s_{x}^{2}=S_{x}^{2}\left(1+e_{1}\right)$ and from the definition of $e_{0}$ and $e_{1}$ we obtain:

$$
\begin{aligned}
& E\left[e_{0}\right]=E\left[e_{1}\right]=0, \quad E\left[e_{0}^{2}\right]=\frac{1-f}{n}\left(\beta_{2(y)}-1\right), \\
& E\left[e_{1}^{2}\right]=\frac{1-f}{n}\left(\beta_{2(x)}-1\right), \\
& E\left[e_{0} e_{1}\right]=\frac{1-f}{n}\left(\lambda_{21}-1\right)
\end{aligned}
$$$$
\hat{S}_{M S i}^{2}=S_{y}^{2}\left(1+e_{0}\right)\left(1-A_{M S i} e_{1}+A_{M S i}^{2} e_{1}^{2}-A_{M S i}^{3} e_{1}^{3}+\ldots . .\right)
$$

Expanding and neglecting the terms more than $3^{\text {rd }}$ order, we get

$\hat{S}_{M S i}^{2}=S_{y}^{2}+S_{y}^{2} e_{0}-S_{y}^{2} A_{M S i} e_{1}-S_{y}^{2} A_{M S i} e_{0} e_{1}+S_{y}^{2} A_{M S i}^{2} e_{1}^{2}$

$\hat{S}_{M S i}^{2}-S_{y}^{2}=S_{y}^{2} e_{0}-S_{y}^{2} A_{M S i} e_{1}-S_{y}^{2} A_{M S i} e_{0} e_{1}+S_{y}^{2} A_{M S i}^{2} e_{1}^{2}$

By taking expectation on both sides of (5.5), we get

$E\left(\hat{S}_{M S i}^{2}-S_{y}^{2}\right)=S_{y}^{2} E\left(e_{0}\right)-S_{y}^{2} A_{M S i} E\left(e_{1}\right)-S_{y}^{2} A_{M S i} E\left(e_{0} e_{1}\right)+S_{y}^{2} A_{M S i}^{2} E\left(e_{1}^{2}\right)$

$\operatorname{Bias}\left(\hat{S}_{M S i}^{2}\right)=S_{y}^{2} A_{M S i}^{2} E\left(e_{1}^{2}\right)-S_{y}^{2} A_{M S i} E\left(e_{0} e_{1}\right)$

The proposed estimator $\hat{S}_{M S i}^{2} ; i=1,2,3, r, d, a$ is given below:

$$
\begin{aligned}
& \hat{S}_{M S i}^{2}=s_{y}^{2}\left[\frac{S_{x}^{2}+\alpha a_{i}}{s_{x}^{2}+\alpha a_{i}}\right]_{(5.1)} \\
& \Rightarrow \quad \hat{S}_{M S i}^{2}=s_{y}^{2}\left(1+e_{0}\right)\left[\frac{S_{x}^{2}+\alpha a_{i}}{s_{x}^{2}+e_{1} S_{x}^{2}+\alpha a_{i}}\right] \Rightarrow
\end{aligned}
$$

$\operatorname{Bias}\left(\hat{S}_{M S i}^{2}\right)=\gamma S_{y}^{2} A_{M S i}\left[A_{M S i}\left(\beta_{2(x)}-1\right)-\left(\lambda_{21}-1\right)\right]$

Squaring both sides of (5.5) and neglecting the terms more than $2^{\text {nd }}$ order and taking expectation, we get

$E\left(\hat{S}_{M S i}^{2}-S_{y}^{2}\right)^{2}=S_{y}^{4} E\left(e_{0}^{2}\right)+S_{y}^{4} A_{M S i}^{2} E\left(e_{1}^{2}\right)-2 S_{y}^{4} A_{M S i} E\left(e_{0} e_{1}\right)$ 
$\operatorname{MSE}\left(\hat{S}_{M S i}^{2}\right)=\gamma S_{y}^{4}\left[\left(\beta_{2(y)}-1\right)+A_{M S i}^{2}\left(\beta_{2(x)}-1\right)-2 A_{M S i}\left(\lambda_{21}-1\right)\right]$

$(5.10)$

\section{Efficiency conditions}

Here, we have derived the efficiency conditions of proposed estimators with other existing estimators under which proposed estimators have performed better than the existing estimators

The bias and Mean square error of existing ratio type estimators up to the first order of approximation is given by

$\operatorname{Bias}\left(\hat{S}_{K}^{2}\right)=\gamma S_{y}^{2} R_{K}\left[R_{K}\left(\beta_{2 x}-1\right)-\left(\lambda_{21}-1\right)\right]$

$\operatorname{MSE}\left(\hat{S}_{K}^{2}\right)=\gamma S_{y}^{4}\left[\left(\beta_{2 y}-1\right)+R_{K}^{2}\left(\beta_{2 x}-1\right)-2 R_{K}\left(\lambda_{21}-1\right)\right]$

$R_{K}=$ Existing. cons $\tan t$

, $K=1,2,3,4 \ldots \ldots$.

Bias, MSE and constant of proposed estimators is given by

$\operatorname{Bias}\left(\hat{S}_{P}^{2}\right)=\gamma S_{y}^{2} R_{P}\left[R_{P}\left(\beta_{2 x}-1\right)-\left(\lambda_{21}-1\right)\right]$

$\operatorname{MSE}\left(\hat{S}_{P}^{2}\right)=\gamma S_{y}^{4}\left[\left(\beta_{2 y}-1\right)+R_{P}^{2}\left(\beta_{2 x}-1\right)-2 R_{P}\left(\lambda_{21}-1\right)\right]$

$R_{P}=$ proposed.cons $\tan t$

$P=1,2,3$

From Equation (6.2) and (6.3), we have

$\operatorname{MSE}\left(\hat{S}_{P}^{2}\right) \leq \operatorname{MSE}\left(\hat{S}_{K}^{2}\right) i f \lambda_{21} \geq 1+\frac{\left(R_{P}+R_{K}\right)\left(\beta_{2 x}-1\right)}{2}$

$\operatorname{MSE}\left(\hat{S}_{P}^{2}\right) \leq \operatorname{MSE}\left(\hat{S}_{K}^{2}\right)$

$\gamma S_{y}^{4}\left[\left(\beta_{2 y}-1\right)+R_{P}^{2}\left(\beta_{2 x}-1\right)-2 R_{P}\left(\lambda_{21}-1\right)\right]_{\leq}$ $\gamma S_{y}^{4}\left[\left(\beta_{2 y}-1\right)+R_{K}^{2}\left(\beta_{2 x}-1\right)-2 R_{K}\left(\lambda_{21}-1\right)\right]$

$\underset{\Rightarrow}{\Rightarrow}\left[\left(\beta_{2 y}-1\right)+R_{P}^{2}\left(\beta_{2 x}-1\right)-2 R_{P}\left(\lambda_{21}-1\right)\right]_{\leq}$
$\left[\left(\beta_{2 y}-1\right)+R_{K}^{2}\left(\beta_{2 x}-1\right)-2 R_{K}\left(\lambda_{21}-1\right)\right]_{(6.6)}$

$\Rightarrow\left[R_{P}^{2}\left(\beta_{2 x}-1\right)-2 R_{P}\left(\lambda_{21}-1\right)\right]_{\leq}$

$\left[R_{K}^{2}\left(\beta_{2 x}-1\right)-2 R_{K}\left(\lambda_{21}-1\right)\right]$

$\Rightarrow\left(\beta_{2 x}-1\right)\left(R_{P}^{2}-R_{K}^{2}\right)\left[-2 R_{P}\left(\lambda_{21}-1\right)\right]_{\leq}$ $\left[-2 R_{K}\left(\lambda_{21}-1\right)\right]_{(6.8)}$

$\Rightarrow\left(\beta_{2 x}-1\right)\left(R_{P}^{2}-R_{K}^{2}\right)\left[-2\left(\lambda_{21}-1\right)\left(R_{P}-R_{K}\right)\right]_{\leq}$ 0 (6.9)

$\Rightarrow\left(\beta_{2 x}-1\right)\left(R_{P}^{2}-R_{K}^{2}\right) \leq\left[2\left(\lambda_{21}-1\right)\left(R_{P}-R_{K}\right)\right]$ (6.10)

$\Rightarrow\left(\beta_{2 x}-1\right)_{\leq} \frac{2\left(\lambda_{21}-1\right)\left(R_{P}-R_{K}\right)}{\left(R_{P}^{2}-R_{K}^{2}\right)}$

$\Rightarrow\left(\beta_{2 x}-1\right) \leq \frac{2\left(\lambda_{21}-1\right)\left(R_{P}-R_{K}\right)}{\left(R_{P}-R_{K}\right)\left(R_{P}+R_{K}\right)}$
$\Rightarrow\left(\beta_{2 x}-1\right)\left(R_{P}+R_{K}\right) \leq 2\left(\lambda_{21}-1\right)$

By solving equation (6.13), we get

$\operatorname{MSE}\left(\hat{S}_{P}^{2}\right) \leq \operatorname{MSE}\left(\hat{S}_{K}^{2}\right)$ if $\lambda_{21} \geq 1+\frac{\left(R_{P}+R_{K}\right)\left(\beta_{2 x}-1\right)}{2}$

\section{Numerical Illustration}

We use the data set of Sarginder Singh; Advance Sampling theory with Applications, page (1135-1136) (source: Agricultural Statistics, 1999 Washington, US) in which Amount (in \$000) is real estate farm loans in different states during 1997 denoted by X (auxiliary variable) and Amount (in \$000) is non-real estate farm loans in different states during 1997 denoted by Y (study variable). 
Table.1 Bias and mean square error of the existing and the proposed estimators

\begin{tabular}{|l|l|l|}
\hline \multicolumn{1}{|c|}{ Estimators } & \multicolumn{1}{c|}{ Bias } & \multicolumn{1}{c|}{ MSE } \\
\hline Isaki (1983) & 10162.12 & 2154636192.33 \\
\hline Kadilar and Cingi (2006a) & 10142.43 & 2147538179.27 \\
\hline Upadhya and Singh (1999) & 10142.43 & 2147538179.27 \\
\hline Proposed (MS1) & 5539.36 & 515446446.37 \\
\hline Proposed (MS2) & 5083.60 & 352496052.61 \\
\hline
\end{tabular}

Table.2 Percent relative efficiency of proposed estimators with existing estimators

\begin{tabular}{|l|l|l|}
\hline Existing Estimators & Proposed estimator $\mathbf{P}_{1}$ & Proposed Estimator $\mathbf{P}_{2}$ \\
\hline Isaki (1983) & 418.01 & 611.25 \\
\hline Kadilar \& Cingi (2006a) & 416.63 & 609.23 \\
\hline Upadhya and Singh (1999) & 416.63 & 609.23 \\
\hline
\end{tabular}

We apply the proposed and existing estimators to this data set and the data statistics is given below:

$\mathrm{N}=50, \quad \mathrm{~S}_{\mathrm{x}}=1084.48, \quad \mathrm{n}=20, \quad \mathrm{Cx}=1.2357$, $\bar{X}=877.558, \quad \beta_{2(x)}={ }_{1.9291}, \quad \bar{Y}=555.434$, $\beta_{2(y)}=0.856227 \quad \rho=0.804 \beta_{1(x)}=1.6624$ $\lambda_{21}=0.9387 \quad \mathrm{~S}_{\mathrm{y}}=584.825, \quad \mathrm{Q}_{2}=452.51$, $\mathrm{Q} 1=57.37, \mathrm{TM}=493.7, \mathrm{Q} 3=1215.67, \mathrm{Q}_{\mathrm{d}}=$ $579.15, \mathrm{Q}_{\mathrm{a}}=636.52, \mathrm{Q}_{\mathrm{r}}=1158.30$.

\section{Results and Discussion}

The modified new improved estimators by utilizing combination of conventional and nonconventional parameters as auxiliary information have performed better than other existing estimators. The improvement can be easily judged from the table 1 and 2 by comparing the bias, MSE and percent relative efficiency of existing and new modified estimators. Hence new modified estimators may be preferred over existing estimators for use in practical applications.

\section{References}

Isaki, C.T. (1983). Variance estimation using auxiliary information. Journal of the American Statistical Association, 78, 117 123.

Kadilar, C. and Cingi, H. (2006a). Improvement in Variance estimation using auxiliary information Hacettepe Journal of mathematics and Statistics, 35(1). 117115.

Upadhya, L. N. and Singh, H. P, (1999): Use of auxiliary variable in the estimation of population variance, Mathematical forum, 4, 33-36 (1936).

Bhat M.A., S. Maqbool, S.A. Saraf, Ab. Rouf and S.H. Malik, NLMID: 101666096. Journal of Advances in Research 13(2): 1-6, 2018 Article no. 37321.

Sarginder Singh; Advance Sampling theory with Applications (2003), page (1135-1136). Publisher; Springer-Science, business.

\section{How to cite this article:}

Bhat, M.A., T.A. Raja and Maqbool, S. 2018. Impact of Robust Estimators on Variance Estimation in Survey Sampling, Using Conventional and Non-Conventional Parameters as Auxiliary Information. Int.J.Curr.Microbiol.App.Sci. 7(10): 3358-3362. doi: https://doi.org/10.20546/ijcmas.2018.710.389 